\title{
Genome mining of Streptomyces ambofaciens
}

\author{
Bertrand Aigle • Sylvie Lautru • Dieter Spiteller • \\ Jeroen S. Dickschat $\cdot$ Gregory L. Challis · \\ Pierre Leblond · Jean-Luc Pernodet
}

\begin{abstract}
Since the discovery of the streptomycin produced by Streptomyces griseus in the middle of the last century, members of this bacterial genus have been largely exploited for the production of secondary metabolites with wide uses in medicine and in agriculture. They have even been recognized as one of the most prolific producers of natural products among microorganisms. With the onset of the genomic era, it became evident that these microorganisms still represent a major source for the discovery of novel secondary metabolites. This was highlighted with
\end{abstract}

\section{B. Aigle $(\triangle) \cdot$ P. Leblond}

Université de Lorraine, Dynamique des Génomes et Adaptation Microbienne, UMR 1128, 54506 Vandœuvre-lès-Nancy, France e-mail: bertrand.aigle@univ-lorraine.fr

B. Aigle $\cdot$ P. Leblond

INRA, Dynamique des Génomes et Adaptation Microbienne, UMR 1128, 54506 Vandœuvre-lès-Nancy, France

S. Lautru - J.-L. Pernodet

Université Paris-Sud, Institut de Génétique et Microbiologie, UMR 8621, Orsay, France

\section{S. Lautru $\cdot$ J.-L. Pernodet}

CNRS, Institut de Génétique et Microbiologie, UMR 8621, Orsay, France

\section{Spiteller}

Department of Biology, Chemical Ecology/Biological Chemistry, University of Konstanz, Universität Strasse 10, 78457 Konstanz, Germany

\section{J. S. Dickschat}

Institute of Organic Chemistry, TU Braunschweig, Hagenring 30, 38106 Braunschweig, Germany

\section{G. L. Challis}

Department of Chemistry, University of Warwick, Coventry CV4 7AL, UK the complete genome sequencing of Streptomyces coelicolor A3(2) which revealed an unexpected potential of this organism to synthesize natural products undetected until then by classical screening methods. Since then, analysis of sequenced genomes from numerous Streptomyces species has shown that a single species can carry more than $30 \mathrm{sec}-$ ondary metabolite gene clusters, reinforcing the idea that the biosynthetic potential of this bacterial genus is far from being fully exploited. This review highlights our knowledge on the potential of Streptomyces ambofaciens ATCC 23877 to synthesize natural products. This industrial strain was known for decades to only produce the drug spiramycin and another antibacterial compound, congocidine. Mining of its genome allowed the identification of 23 clusters potentially involved in the production of other secondary metabolites. Studies of some of these clusters resulted in the characterization of novel compounds and of previously known compounds but never characterized in this Streptomyces species. In addition, genome mining revealed that secondary metabolite gene clusters of phylogenetically closely related Streptomyces are mainly species-specific.

Keywords Genome mining · Natural products . Biosynthetic pathways $\cdot$ Streptomyces ambofaciens

\section{Introduction}

The emergence of the genomics era during the last decade has given a fresh boost in the field of drug discovery from microbial natural products (also called secondary metabolites). Before what can be called a "revolution" in this field, approaches for searching for new bioactive molecules were mainly based on (high-throughput) screening of extracts of potential producers either by 
compound-guided or bioactivity-guided approaches [70]. Nevertheless, discovery of new molecules by methods such as the bioactivity-guided approach has shown limitations over the years, partly because of a problem of recurrence, i.e. the re-isolation of already known compounds. For example, streptothricin and streptomycin are respectively found in about 10 and $1 \%$ of randomly collected soil actinomycetes, which are among the most prolific microorganisms producing natural products [4]. It was thus believed that microorganisms were an exhausted source of natural products and this is one of the reasons for the withdrawal of many pharmaceutical companies from the natural product drug discovery programmes.

In recent years, however, genome sequencing has revealed that microorganisms still represent an important source for novel natural products by disclosing a so far hidden secondary metabolome. In the case of the bacteria of the Streptomyces genus, which are the topic of this review, the analysis of the first completely sequenced genome, the genome of Streptomyces coelicolor A3(2), has indeed revealed an unsuspected and unprecedented potential to synthesize secondary metabolites. Indeed, whereas only five metabolites were identified over 50 years by classical screening approaches, genome analysis has shown that $S$. coelicolorA3(2) had the capability to produce nearly 20 additional natural products [7]. Since then, several of these compounds have been characterized like the tetrapeptide iron chelator coelichelin [38] or the novel polyketide alkaloid coelimycin P1 synthesized by a cryptic type I PKS gene cluster [20]. This situation is not specific to $S$. coelicolor A3(2). Genome sequencing (complete or partial) of numerous Streptomyces species has indeed shown that they all contain a large number of cryptic secondary metabolite gene clusters (up to 30 , sometimes more). In addition, thanks to the genome mining, several biosynthetic gene clusters of previously known compounds have been identified such as the cluster responsible for the production of the siderophores desferrioxamines [6].

The large number of secondary metabolite gene clusters compared to the number of metabolites known to be produced by a single strain is usually explained by the fact that some of these gene clusters are cryptic, i.e. not expressed or the products are formed at a level too low to be detected in laboratory growth conditions. This represents the major bottleneck to get access to potentially novel compounds. Several methods have been developed to activate these silent gene clusters, such as overexpression of a pathway-specific activator, manipulation of global transcriptional regulators, mutation in the RNA polymerase subunits, ribosome engineering, uses of histone deacetylase inhibitors, cultures in different growth conditions or heterologous expression in a host engineered for expression. These methods have been described in different reviews (see in this issue [22, 53, 58, 75] and for example [2, 21, 48, 52, 69]) and will therefore not be presented here.

Streptomyces ambofaciens ATCC 23877 is an industrial strain isolated in the early 1950s [56] and exploited for the production of the macrolide spiramycin, which is used in human medicine as an antibacterial agent and for the treatment of Toxoplasma infections. In $1952 \mathrm{~S}$. ambofaciens ATCC 23877 was also discovered to produce another antibiotic, the pyrrolamide congocidine, which is too cytotoxic for clinical uses. The 16S RNA sequence analysis revealed that $S$. ambofaciens ATCC 23877 is very closely related to $S$. coelicolor A3(2) (only $1.1 \%$ divergence) [13]. Pairwise genome comparison revealed a high level of synteny between the two species, except for two inversion events centred on the origin of replication. The divergence clusters in the terminal regions of the linear chromosome [13]. Nevertheless, as presented below, more than half (14) of the 25 secondary metabolite gene clusters identified in S. ambofaciens ATCC 23877 (Fig. 1) are not conserved in S. coelicolor A3(2) and reciprocally. And interestingly, among the natural products synthesized by each species, the specificity concerns mainly the arsenal of antibiotics. Among the shared clusters, some have already been characterized in either one or both species, including clusters responsible for the biosynthesis of siderophores (desferrioxamines and coelichelin), of volatile terpenoids [geosmin, 2-methylisoborneol (MIB), albaflavenone] or of carotenoids (already described in [1]). Altogether, this showed that although closely related to $S$. coelicolor, $S$. ambofaciens represents a source for novel bioactive secondary metabolites.

\section{Analysis of genome mined secondary metabolite gene clusters}

Identification of the congocidine cluster and full characterization of the spiramycin cluster

As described above, S. ambofaciens ATCC 23877 was known to produce two antibacterial compounds, the macrolide spiramycin and the pyrrolamide congocidine [56]. However, the cluster responsible for the production of congocidine had never been identified and the one involved in the biosynthesis of spiramycin was only partially characterized [17, 60] prior to the onset of the $S$. ambofaciens genome sequencing. Mining the genome of this strain allowed the identification and full characterization of these clusters. In addition, it revealed the original biosynthetic pathway of the pyrrolamide antibiotic. 


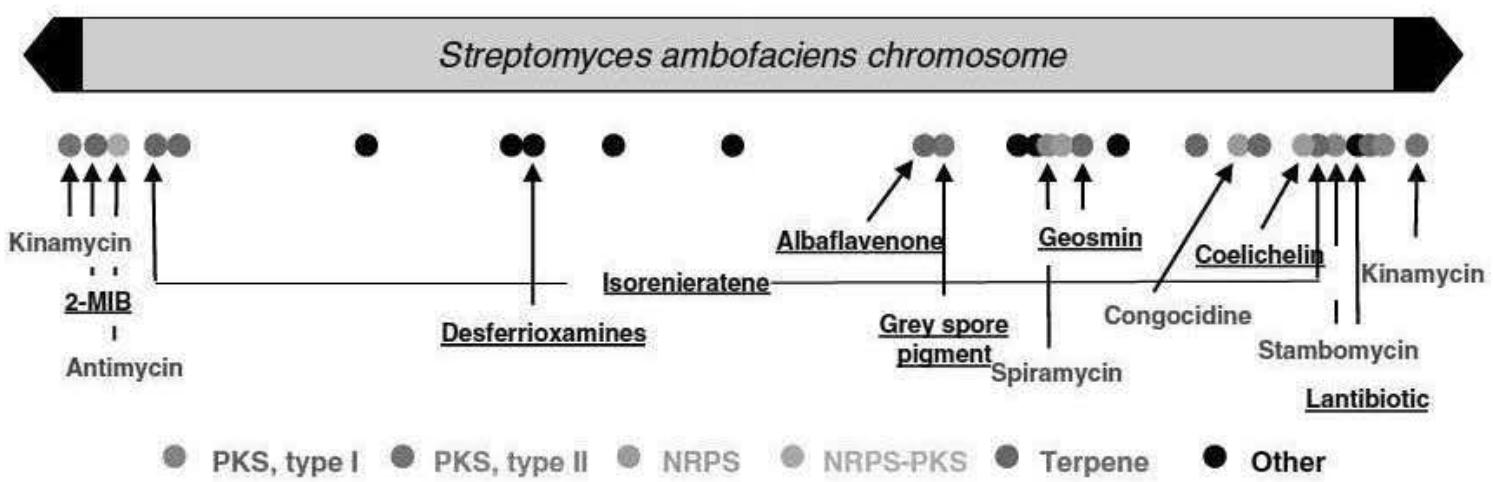

Fig. 1 Schematic representation of the linear chromosome of $S$. ambofaciens ATCC 23877 and location of the secondary metabolite gene clusters identified so far. Clusters are indicated by filled circles. The colours correspond to the type of genes found in the cluster.

Metabolites common to $S$. ambofaciens and $S$. coelicolor are underlined. Metabolites specific to $S$. ambofaciens are in blue. The black arrows at the extremities of the chromosomal arms symbolize the terminal inverted repeats (TIRs)

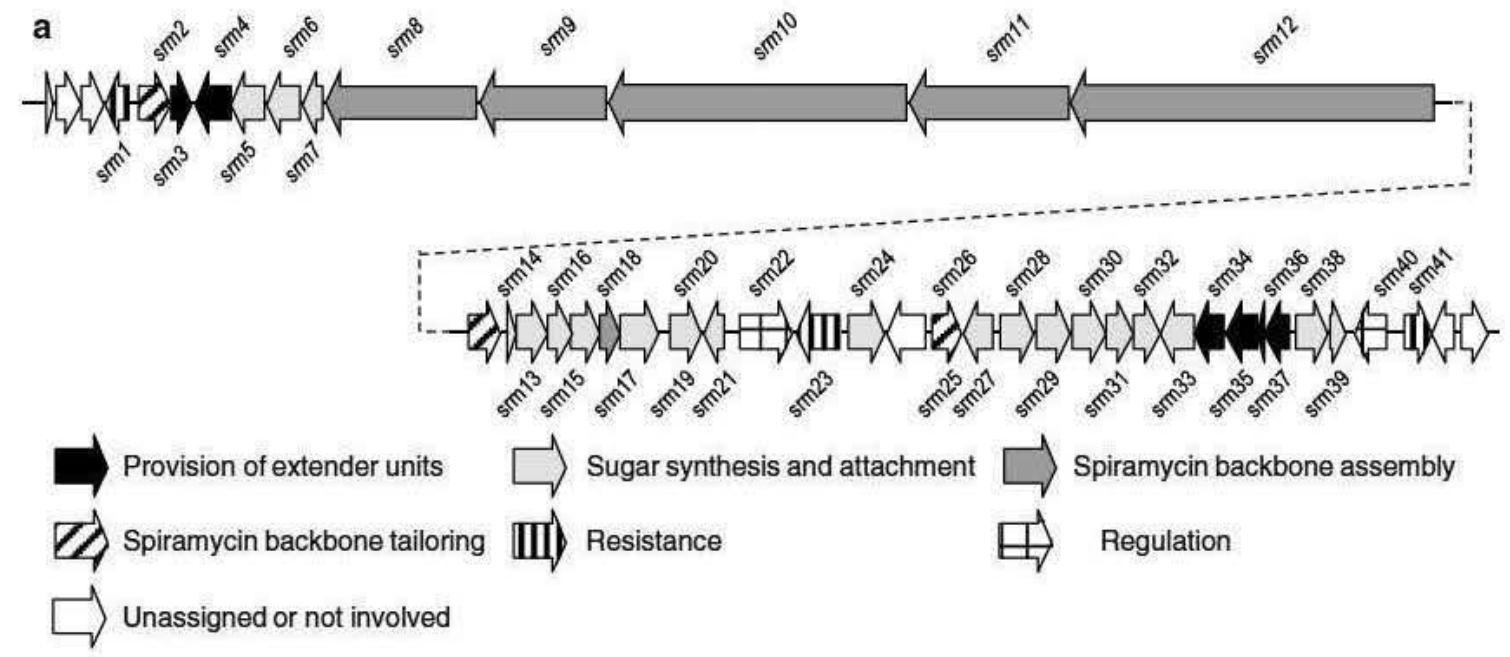

b

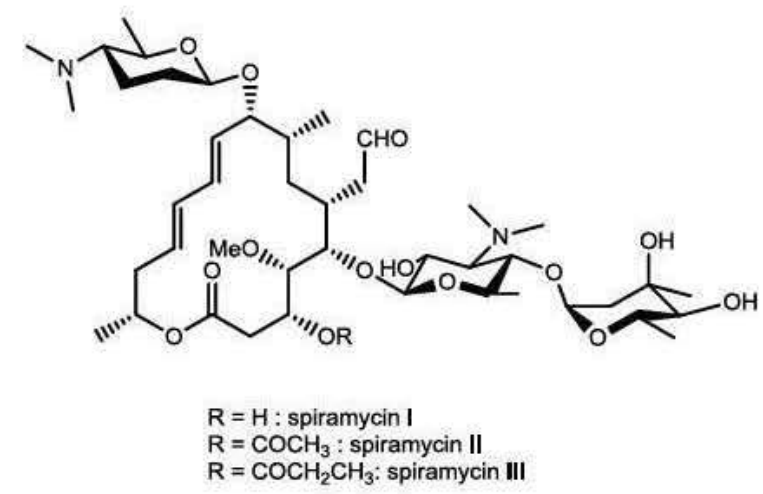

Fig. 2 Streptomyces ambofaciens ATCC 23877 spiramycin biosynthetic gene cluster. a Genetic organisation of the spiramycin gene cluster. b Chemical structure of spiramycin

\section{Spiramycin}

Spiramycins I, II and III are 16-membered ring macrolide antibiotics used in human therapeutics as antibacterial and antiparasitic agents (active against Toxoplasma spp.) [12,
57]. They comprise a core macrolactone ring (platenolide I), to which two amino sugars (mycaminose, forosamine) and one neutral sugar (mycarose) are attached (Fig. 2).

Spiramycin biosynthesis has been studied for more than 30 years and the gene cluster directing this biosynthesis 
(srm cluster) was isolated prior to $S$. ambofaciens ATCC 23877 genome sequencing [30, 60]. Involvement of this gene cluster in spiramycin production has been established by inactivating several genes $[30,60]$. The srm cluster comprises 41 genes spanning an $84-\mathrm{kb}$ region. Among these genes, 36 are involved in spiramycin biosynthesis, two are responsible for spiramycin biosynthesis regulation and three confer spiramycin resistance.

The spiramycin macrolactone backbone is synthesized by a PKS enzyme comprising five subunits ( $\mathrm{srm} 8$ to $\mathrm{srm} 12$ ) organized in 8 modules and 36 domains, with the ketoreductase domain of module 4 probably being inactive [34]. Apart from the classical malonyl-CoA and methylmalonylCoA precursors, this PKS also incorporates ethylmalonylCoA and methoxymalonyl-CoA. Enzymes involved in the biosynthesis of these extender units are encoded within the $s r m$ cluster: Srm4, a putative crotonyl-CoA reductase probably participates in the synthesis of ethylmalonyl-CoA and Srm3, Srm34, Srm35, Srm36 and Srm37 are likely involved in methoxymalonyl-acyl carrier protein biosynthesis (Fig. 2).

After the biosynthesis of the macrolactone ring (platenolide I), this intermediate undergoes several post-PKS modifications, including oxidation of the $\mathrm{C}_{19}$ methyl group, the reduction of the $\mathrm{C}_{9}$ keto group, the attachment of the mycaminose, forosamine and mycarose sugars, and the acylation of the $\mathrm{C}_{3}$ hydroxyl group to yield spiramycin II and III. All genes required for the synthesis of the three sugars mycaminose, forosamine and mycarose from glucose-1-phosphate are present in the spiramycin gene cluster. We recently identified the genes involved in most post-PKS tailoring steps $[49,50]$. On the basis of gene deletions and LC-MS-MS analyses, we proposed the following timeline for these various steps: platenolide I is first reduced at the $\mathrm{C}_{9}$ position by Srm26. The resulting molecule (platenolide II) is then glycosylated by Srm5 (addition of mycaminose). The $\mathrm{C}_{19}$ methyl is subsequently oxidized by Srm13. The successive additions of forosamine and mycarose are next catalysed by the Srm29 and Srm38 glycosyltransferases, respectively. Acylation of the $\mathrm{C}_{3}$ hydroxyl group of spiramycin I, yielding spiramycins II and III, is most likely the last step of the synthesis and catalysed by Srm2. Two out of three glycosyltransferases involved in spiramycin biosynthesis, Srm5 and Srm29, require auxiliary proteins for their activity. The auxiliary protein Srm6 interacts only with Srm5 (mycaminosyltransferase) whereas the other one, Srm28, interacts efficiently with Srm5 and Srm29 (forosaminyltransferase) [50].

Two srm genes are involved in resistance to spiramycin, srm1 and srm23. Srm1 (formerly SrmD) is a $23 \mathrm{~S}$ rRNA methyltransferase (unpublished results) preventing the binding of spiramycin to its ribosomal target and Srm 23 (formerly $\mathrm{SrmB}$ ) is the cytoplasmic ATP-binding component of an ABC transporter [61]. A third gene, srm41, encodes a putative glycosidase that may be involved in spiramycin reactivation.

Two srm genes, $s r m 22$ (formerly $s r m R$ ) and $s r m 40$, are involved in the regulation of spiramycin biosynthesis. Srm22 is a transcriptional activator controlling the transcription of Srm40. Srm40, a homologue of the tylosin transcriptional activator TylR, is the pathway-specific activator that controls the expression of all srm genes except for $\operatorname{srm} 22$ and $\operatorname{srm} 25$ [17, 29].

\section{Congocidine}

Congocidine (also called netropsin) belongs to the family of pyrrolamide antibiotics characterized by a 4-aminopyrrole-2-carboxyl moiety. Congocidine was first discovered in 1951 by Finlay et al. [16] from a culture extract of Streptomyces netropsis. Shortly after, in 1952, Cosar et al. [15] reported its production by $S$. ambofaciens. Members from the pyrrolamide family, which includes distamycin, kikumycins or pyrronamycins, bind non-covalently (except for pyrronamycin $\mathrm{B}$, which probably binds covalently) into the minor groove of DNA with some sequence specificity (a succession of 4 or more $\mathrm{A} / \mathrm{T}$ bases). This endows them with biological activities such as antibacterial, antiviral or antitumour activities, but also renders them too toxic for any therapeutic use.

Although the chemical characterization of the first members of the pyrrolamide family was described almost 60 years ago, no pyrrolamide biosynthetic gene cluster or pathway was known when we reported the identification and characterization of the congocidine biosynthetic gene cluster (cgc) in S. ambofaciens in 2009 [26]. The $c g c$ cluster is located in one of the variable extremities of the S. ambofaciens chromosome (Fig. 1), in a genomic island specific to $S$. ambofaciens situated in a region otherwise syntenic with the chromosome of $S$. coelicolor which does not produce congocidine. The $c g c$ gene cluster is composed of 22 genes ( $c g c 1$ to $c g c 22$ ) (Fig. 3), comprising one regulatory gene $(\operatorname{cgcl})$ and two resistance genes $(\operatorname{cgc} 20$ and $c g c 21$ ), encoding the two subunits of an $\mathrm{ABC}$ transporter homologous to NetP1 and NetP2 conferring congocidine resistance in $S$. netropsis [65]. The 19 remaining $\operatorname{cgc}$ genes are involved in the biosynthesis of the precursors and in the assembly of the molecule.

Three precursors have been identified for the biosynthesis of congocidine: guanidinoacetate, 3-aminopropionamidine and 4-acetamidopyrrole-2-carboxylate. This last precursor, common to all pyrrolamides, is synthesized from $\mathrm{N}$-acetylglucosamine-1-phosphate. Its biosynthesis involves several carbohydrate processing enzymes and constitutes the first example of a pyrrole moiety derived from 

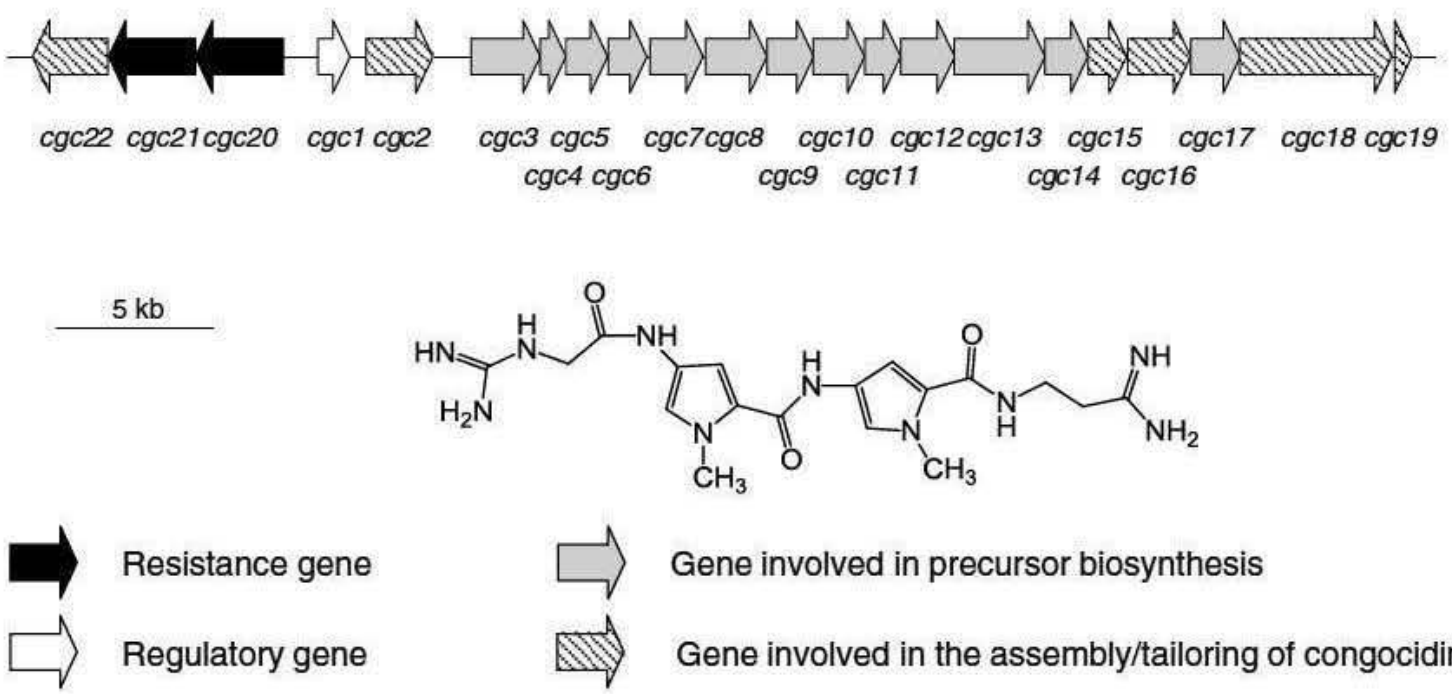<smiles>Cn1cc(NC(=O)CNC(=N)N)cc1C(=O)NCCC(=N)N</smiles>

Gene involved in precursor biosynthesis

Gene involved in the assembly/tailoring of congocidine

Fig. 3 Streptomyces ambofaciens ATCC 23877 congocidine biosynthetic gene cluster. a Genetic organisation of the congocidine gene cluster. b Chemical structure of congocidine

a carbohydrate molecule [40]. The biosynthetic pathways of the two other precursors, guanidinoacetate and 3-aminopropionamidine, remain to be established.

Congocidine is assembled by an atypical nonribosomal peptide synthetase (NRPS) comprising one free-standing module ( $\mathrm{Cgc18}$ ) and four single-domain proteins (two condensation domains, $\mathrm{Cgc} 2$ and $\mathrm{Cgc} 16$, one peptidyl carrier protein $\mathrm{Cgc19}$ and an acyl-CoA synthetase $\mathrm{Cgc} 22$ ). Although unusual, this NRPS architecture appears to be well suited for the polymeric assembly of 4-aminopyrrole-2-carboxylic acid occurring during the biosynthesis of pyrrolamides (for a congocidine biosynthetic assembly model, see [26]). This arylamine pyrrole precursor is probably toxic for the cell and is not observed free in solution in a congocidine assembly impaired mutant, contrary to 4-acetamidopyrrole-2-carboxylate. Thus, 4-acetamidopyrrole-2-carboxylate is probably loaded onto the NRPS and deacetylation occurs on the PCP-bound molecule, before condensation with the other substrates can take place.

New secondary metabolites identified from genome mining

Analysis of the S. ambofaciens ATCC 23877 genome sequence highlighted the presence of 14 secondary metabolite gene clusters specific to $S$. ambofaciens ATCC 23877 , or at least not conserved in the closely related species $S$. coelicolor A3(2). Within these clusters, 10 could not be associated with any product or a predicted product. Study of two of these clusters allowed the identification of new compounds produced by $S$. ambofaciens including a novel macrolide with promising antiproliferative activities.

\section{Kinamycins}

Among the clusters identified from genome sequence analysis, a type II PKS gene cluster located within the 200-kblong terminal inverted repeats (TIRs) of the chromosome (Fig. 1), a structure characteristic of the linear replicons in Streptomyces, suggested that S. ambofaciens ATCC 23877 had the potential to synthesize an additional antibiotic besides spiramycin and congocidine. This duplicated cluster, named alp cluster (see below, Fig. 4), covers a region of $30 \mathrm{~kb}$ and encompasses $27 \mathrm{ORFs}$ and was predicted to be responsible for the biosynthesis of an aromatic polyketide belonging to the angucyclinone family (the name alp comes from angucyclinone-like polyketide) [54]. The alp cluster was not expected to govern the biosynthesis of an angucycline antibiotic because it does not contain genes involved in the biosynthesis or the transfer of sugar moieties (angucyclinones are sugarless compounds in contrast to angucyclines). In addition, the region of 12 genes spanning from alpL2 to alpG and including the alpABC locus which encodes the minimal PKS [including three subunits, a $\beta$-ketosynthase (KS), a chain length factor (CLF) and an acyl carrier protein (ACP), and responsible for the assembly of the polyketide chain] is syntenic with the partially characterized kin cluster of Streptomyces murayamaensis responsible for the biosynthesis of the kinamycin angucyclinone antibiotics ([24], accession number AY228175).

The alp cluster was shown to be transcriptionally active under standard growth conditions [54]. The identification of the product(s) of the alp gene cluster was based on deletion of the minimal PKS genes and comparison of the mutant strain with the wild-type strain for their antibacterial activities under different growth conditions. On a 

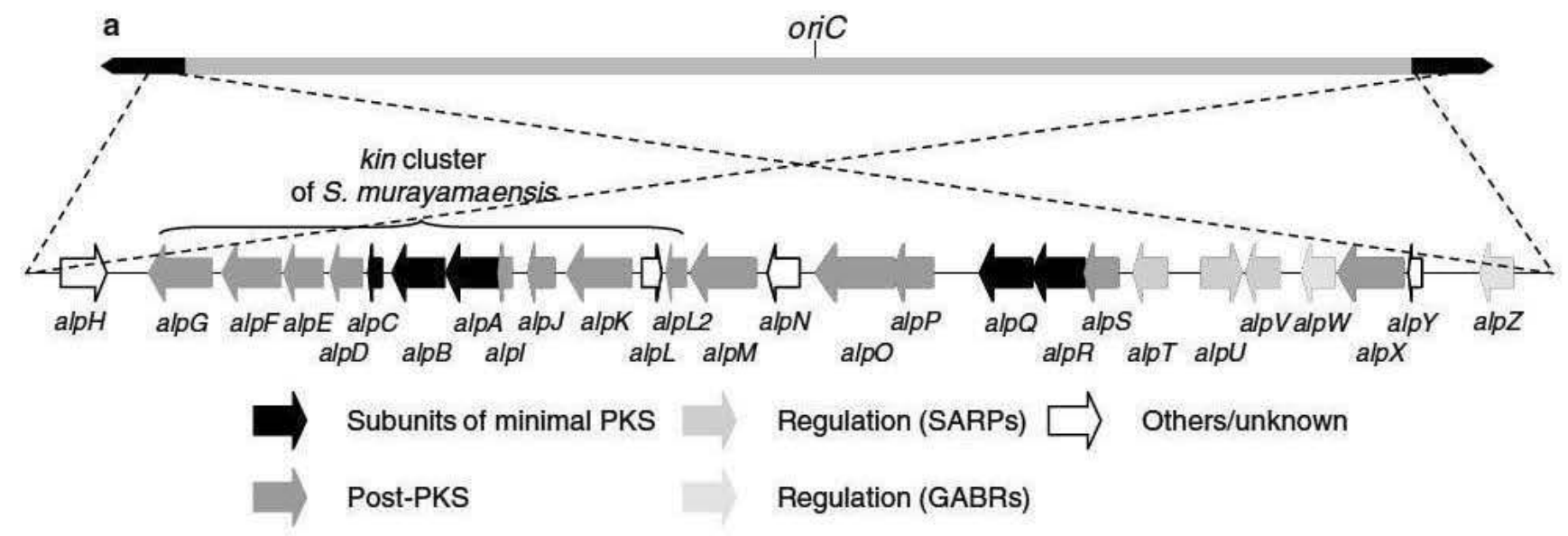

Regulation (SARPs) $\square$ Others/unknown

Regulation (GABRs)

b

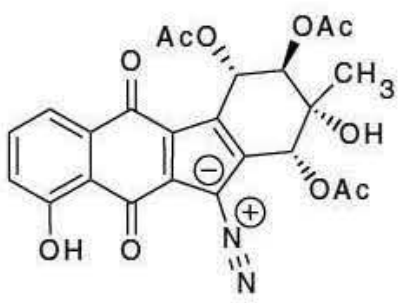

Kinamycin C<smiles></smiles>

Kinamycin D<smiles></smiles>

Epoxy-kinamycin FL-120B'
Fig. 4 Streptomyces ambofaciens ATCC 23877 kinamycin biosynthetic gene cluster. a Chromosomal location and genetic organisation of the kinamycin biosynthetic gene cluster. The black arrows at the extremities of the chromosomal arms symbolize the TIRs. The part of the cluster showing synteny with the kinamycin gene cluster of S. murayamaensis species is indicated. b Chemical structures of the kinamycins identified in S. ambofaciens ATCC 23877

these genes, alpW encodes a product acting as a key late repressor of the cellular control of the production of the antibacterial compound and orange pigment. Indeed, AlpW accumulates in the cell during the phase of antibiotic production and then represses in particular the expression of $a l p V$, an essential pathway-specific activator required for activation of the alp structural genes [3], thus turning off the expression of the biosynthetic genes [11]. In fact, the deletion of the two copies of alpW led to the deregulation of the regulatory pathway and to a mutant strain which persisted in antibiotic production after initial onset of its biosynthesis. Consequently, this strain allowed purification of sufficient material for structure elucidation. Three angucyclinones were purified and identified as members of the kinamycin family [23]: the kinamycin C, D and the epoxy-kinamycin FL120B' [11]. These diazo-substituted benzo[b]fluorene antibiotics are particularly interesting as potential anticancer agents $[25,51]$ and therefore the access to the first complete kinamycin gene cluster will provide the opportunity to generate new derivatives with improved antitumour activity by genetic manipulation.

In addition to the discovery of $S$. ambofaciens ATCC 23877 as a kinamycin producer, the genome mining highlights an original architecture of the alp cluster. This type II 
PKS gene cluster contains two loci encoding minimal PKS, one complete (alpABC encoding respectively a KS, CLF and ACP subunit) and one incomplete (alpRQ, encoding respectively a KS and CLF subunit) which lacks an ACP encoding gene [54] (Fig. 4). Deletion of the alpR and alpQ genes revealed that their products are not involved in the biosynthesis of kinamycins or the orange pigment. AlpR and AlpQ, in association with an ACP, e.g. AlpC, could be responsible for the biosynthesis of another secondary metabolite. Interestingly, the two sets of genes are under the control of the same regulatory system. The alp cluster could thus be considered as a fusion of two clusters that would also share their tailoring enzymes.

\section{Stambomycins}

Among the $S$. ambofaciens ATCC 23877 secondary metabolite gene clusters unveiled by genome mining, a large type I PKS gene cluster located in the right arm of the chromosome at about $500 \mathrm{~kb}$ from the chromosomal end attracted our attention (Figs. 1, 5). Indeed, in silico analysis revealed that this gene cluster could be involved in the production of a novel macrolide and macrolides are at the origin of very important drugs used in human therapy, for example as antibacterial agents (e.g. erythromycin or spiramycin), as immunosuppressors (e.g. rapamycin) or as antifungal agents (e.g. nystatin). Some macrolides also represent a new class of anticancer drugs and compounds such as epothilones and their derivatives are already on the market or in phase II or III clinical trials [27].

The first characteristic of this $S$. ambofaciens type I PKS gene cluster is its particularly large size: it spans about $150 \mathrm{~kb}$ and is in fact one of the largest type I PKS gene clusters ever described. The cluster contains no less than 25 genes (from samR0487 to samR0465) including nine PKS genes and 16 additional genes that flank and are interspersed within the PKS genes and encode proteins predicted to be involved in PKS substrate supply, postand on-PKS tailoring reactions, deoxysugar biosynthesis, regulation and resistance (Fig. 5). The number of modules (25) identified within the PKSs and the number of enzymatic domains (112) identified within these modules using SEARCHPKS [74] are also particularly high. All the enzymatic domains were predicted to be functional except the last ketoreductase (KR) domain in module 24 (Fig. 5; two essential amino acids of the catalytic triad, a tyrosine residue and an asparagine residue, are absent [59]). These data suggested the ability of S. ambofaciens ATCC 23877 to synthesize a molecule of high molecular weight. The order in which the nine PKSs act in polyketide chain assembly was deduced by the identification of the loading module in SamR0467 (presence of a ketosynthase ${ }^{\mathrm{Q}}$ domain at the $\mathrm{N}$ terminus; [9]), the identification of the termination module in SamR0474 (presence of a thioesterase domain at the C terminus; [19]) and by the assumption that the order of the seven other PKSs followed the order of their respective genes in the cluster. Analysis of the AT domains within each module suggested that the product of the PKS was assembled from 16 molecules of malonyl-CoA, eight molecules of methylmalonyl-CoA and one molecule of an unknown extender unit (loaded by the AT13 domain) [73, 74]. In addition, sequence analysis of the ketoreductase and enoylreductase domains allowed the prediction of the stereochemistry of each stereocentre of the polyketide intermediate produced by the PKSs $[31,36]$. All together, these data allowed us to propose a structure of the final PKS-bound intermediate (Fig. 5) with the molecular formula $\mathrm{C}_{61} \mathrm{H}_{107} \mathrm{O}_{18}$. Searches in chemical databases indicated that it was likely to be novel [37]. The final product of this large type I PKS gene cluster was in fact predicted to be a glycosylated lactone. Indeed, on the basis of the thioesterase domain, the polyketide intermediate was expected to be cyclized. The presence of five genes in the cluster homologous to spiramycin biosynthetic genes that are responsible for the synthesis of mycaminose (Fig. 5; [50]) and of a gene (samR0481) encoding a putative glycosyltransferase that could transfer, according to the analysis with SEARCHGTr [28], a mycaminosyl residue to hydroxyl groups in the product of the PKS, indicated that the final product would be glycosylated. The three other biosynthetic genes encoded putative cytochrome P450 (samR0478 and samR0479) and a putative type II thioesterase (samR0475) and were expected to catalyse post-PKS hydroxylation reactions and hydrolysis of aberrant acyl groups attached to the ACP domains of the PKS, respectively $[33,55]$.

Unlike the alp cluster, the genes of the type I PKS gene cluster were silent under standard culture conditions [37]. Therefore, a method to specifically activate expression of this cryptic cluster was developed in order to get access to the potential novel macrolide. This method was based on the overexpression of a gene expected to encode a pathway-specific regulator (heterologous expression approach was excluded at that time because of the large size of the cluster). Three genes encoding putative transcriptional regulators are located within the type I PKS gene cluster. Two of them, samR0468 and samR0469, code for a two-component signal transduction system, with samR0468 coding for a response regulator and samR0469 for a histidine kinase. The product of samR0484 is a member of the LAL (Large ATP-binding LuxR) family. Since members of this family of regulators had already been described as activators of macrolide biosynthesis, such as PikD of Streptomyces venezuelae and $\mathrm{RapH}$ in Streptomyces hygroscopicus, which activate pikromycin and rapamycin production, respectively [35, 72], we overexpressed the expected pathwayspecific regulatory gene samR0484 in the S. ambofaciens 
a
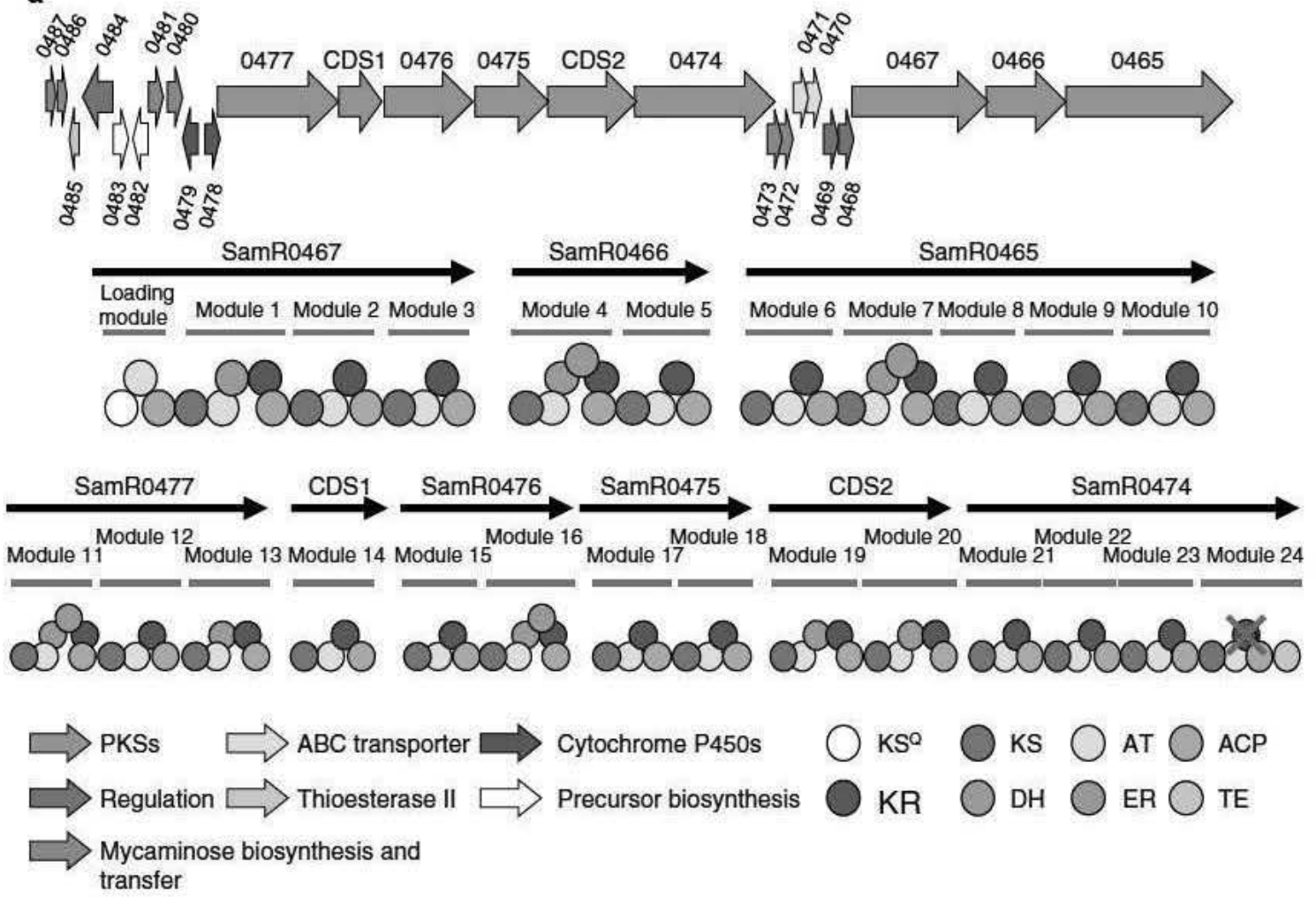

b

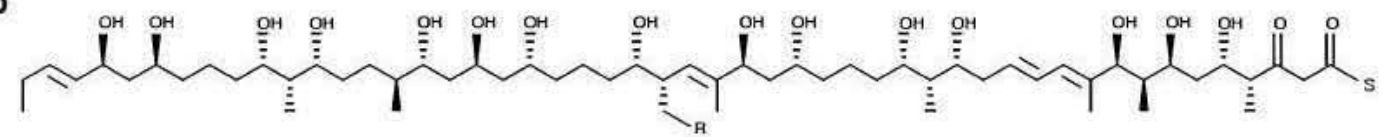

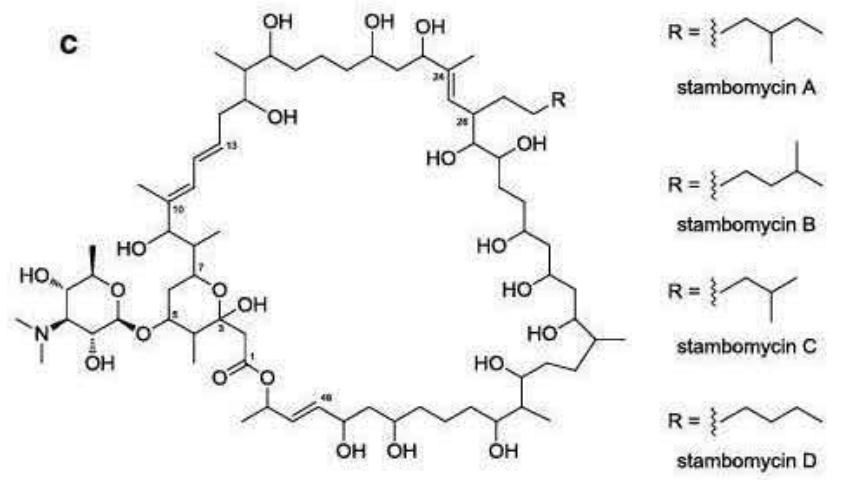

Fig. 5 Streptomyces ambofaciens ATCC 23877 stambomycin biosynthetic gene cluster. a Genetic organisation of the stambomycin gene cluster and module and domain organization of the PKSs encoded by the cluster. Note that the ketoreductase domain in module 24 is predicted to be nonfunctional and is crossed out. $A C P$ acyl carrier protein, $A T$ acyltransferase, $D H$ dehydratase, $E R$ enoylreductase, $K R$

ATCC 23877 wild-type strain. The samR0484 gene was placed under the control the strong and constitutive promoter $e r m E p^{*}$ in the integrative and conjugative vector pB139 [71]. The presence of the recombinant plasmid in ketoreductase, $K S$-ketoacyl synthase, $K S^{Q}, \beta$-ketoacyl synthase in which the active site cysteine residue is replaced by glutamine, TE thioesterase. b Predicted structure of the fully assembled polyketide chain attached to the ACP domain in the last module of the PKS. Note that the structure of the side chain at $\mathrm{C}_{26}$ could not be predicted. c Chemical structures of stambomycins A-D

wild-type $S$. ambofaciens triggered the expression of all the silent genes within the cryptic type I PKS gene cluster.

Comparative metabolic profiling using LC-MS between the strain with ectopic expression of the LAL regulator and 
the control strain (i.e. S. ambofaciens ATCC 23877 with the vector $\mathrm{pIB} 139$ ) revealed two peaks present only in the LAL regulator overexpressing strain [37]. Interestingly, these peaks were only detected from the mycelium extract of this strain but not from the supernatant extract, indicating that, at least in the growth conditions used, the corresponding metabolites were not secreted. Structural characterization of these new natural products was performed by mass spectrometry and NMR. In total, four forms of a 51-membered macrolide which was named stambomycin were characterized (isomers A, B, C and D; Fig. 5) [37].

Stambomycins are the first metabolic products identified in actinomycetes by overexpressing of a pathway-specific regulatory gene and activating a silent gene cluster. A similar strategy was initially and successfully applied in the fungus Aspergillus nidulans and led to the discovery of unique PKS-NPRS metabolites, the aspirydones A and B [8]. Interestingly, identification of LAL regulator genes in numerous cryptic biosynthetic gene clusters in actinomycetes indicate that our approach can be applied as a general strategy for discovery of novel natural products [37].

The study of the stambomycin biosynthesis also revealed two originalities [37]. First, the large lactone ring of stambomycins, resulting from the $\mathrm{C}_{1} / \mathrm{O}_{50}$ ester linkage, is likely due to a novel type of cyclization involving a cytochrome P450-catalysed hydroxylation of the polyketide chain rather than ketoreduction during chain assembly. Second, the hexyl or heptyl substituents at $\mathrm{C}_{26}$ are due to the loading by the AT domain of PKS module 12 of very unusual extender units (hexyl/heptylmalonyl-CoA) onto the ACP domain of this module.

Finally, genome mining of S. ambofaciens ATCC 23877 allowed the discovery of promising new molecules for anticancer therapy. Indeed, the stambomycins showed significant antiproliferative activities against various human cancer cell lines [37].

\section{Antimycins and related volatiles}

The respiratory chain inhibitors antimycins are strong antifungal agents produced by several Streptomyces species. These compounds have been known since the end of the 1940 s when they were identified as highly active compounds against the phytopathogen fungus Venturia inaequalis [41]. Although the structure of these antifungals was solved 40 years ago, nothing was known about the genes responsible for their biosynthesis until recently. Similarly to congocidine, the antimycin biosynthetic gene cluster was identified thanks to a genome mining approach although in a different way. A Streptomyces mutualist associated with attine ants, Streptomyces $\mathrm{S} 4$, was shown to produce the antifungals candicidin and antimycins (eight antimycin compounds, including antimycins $\mathrm{A}_{1}-\mathrm{A}_{4}$, were isolated from this species [64]). On the basis of their structures, antimycins were expected to be at least partially synthesized by an NRPS and genome analysis of Streptomyces S4 identified a hybrid NRPS-PKS cluster predicted to be responsible for the biosynthesis of antimycins. As expected, disruption of this cluster abolished the production of antimycins [64]. Interestingly, blast analysis revealed that it was conserved in two other Streptomyces species, Streptomyces albus J1074 and S. ambofaciens ATCC 23877, in which the cluster is located in the left chromosomal arm and spans from samL0362 to samL0378 (Figs. 1, 6). This strongly suggested that these species also had the potential to produce these antifungals [64].

Streptomyces ambofaciens ATCC 23877 was indeed shown by $\mathrm{LC}-\mathrm{MS}$ analysis to produce antimycins $\mathrm{A}_{1}$ to $\mathrm{A}_{4}$ and deletion of a locus of five genes (samL0365samL0369) within the cluster has confirmed its implication in the biosynthesis of the antifungals [63]. Studies have been also carried out on the biosynthesis of the rare 3-aminosalicylate starter unit of the NRPS. This biosynthesis involves the samL0365-samL0369 locus which encodes a protein complex similar to the multicomponent PaAABCDE oxygenase complex, which catalyses the oxidative ring opening of phenylacetyl-CoA. The samL0365samL0369 locus has indeed been proven to be involved in the synthesis of the starter unit from tryptophan, via anthraniloyl-CoA, which undergoes an unprecedented oxidation and 1,2-shift of its carboxylic acid CoA moiety, likely via an epoxide intermediate [63].

Interestingly, the study of the antimycin cluster in $S$. ambofaciens ATCC 23877 revealed that it was also responsible for the presence of the volatile compounds blastmycinones and butenolides [62]. It was known for a long time that antimycins can easily undergo base-catalysed degradation to blastmycinone derivatives and to $N$-(3-formamido-2-hydroxybenzoyl)-L-threonine [66-68]. In addition, blastmycinones can form either butanolide compounds by deacylation or butenolide volatiles by elimination of a carboxylic acid [18]. Investigation by GC-MS of the volatiles released by $S$. ambofaciens ATCC 23877 revealed the presence of different butenolide and blastmycinone derivatives in the headspace extracts [62]. The structure of the blasmycinones was shown to coincide nicely with the main antimycins identified in this strain. Similarly, the butenolides found amongst the volatiles matched the structure of the blastmycinones produced by the strain [62]. Thus, this strongly suggested that these two types of volatiles are derived from the antimycin biosynthetic pathway (Fig. 6). This hypothesis was confirmed by GC-MS analysis of the headspace extract of the $S$. ambofaciens mutant strain deleted for the samL0365-samL0369 locus. Indeed, in addition to being unable to produce antimycins, the mutant was defective in the production of the blastmycinone and 


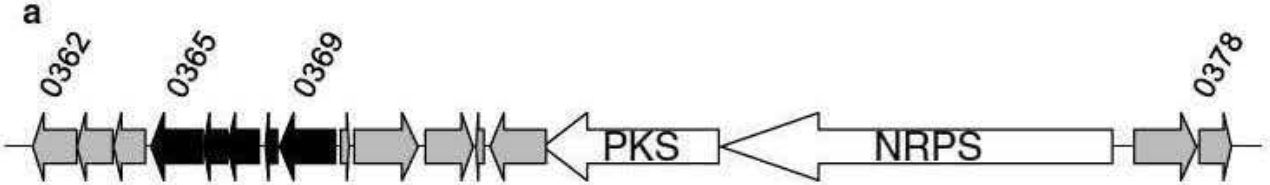<smiles>[R]C(=O)O[C@@H]1[C@H]([2H])C(=O)O[C@@H]1C</smiles>

Fig. 6 Streptomyces ambofaciens ATCC 23877 antimycin biosynthetic gene cluster. a Genetic organisation of the antimycin gene cluster. The dark arrows correspond to the locus samL0365-samL0369 responsible for the biosynthesis of the unusual 3-aminosalicylate

butenolide derivatives that were found in the wild-type strain [62]. It should be noted that it was the first time that butenolides were shown to derive from degradation of antimycin compounds. The biological roles of these volatiles for the producer strain are still unknown.

\section{Discussion}

Access to the complete genome sequence allowed us to identify the potential of S. ambofaciens ATCC 23877 to synthesize natural products. Like in most of its congeners, more than 20 secondary metabolite gene clusters are encoded within the chromosome. S. ambofaciens does not contain any mobile genetic element with such clusters like the linear megaplasmids pSLA2-L of Streptomyces rochei and pSCL4 of Streptomyces clavuligerus which are densely packed with large numbers of gene clusters for secondary metabolites $[46,47]$. The ongoing exploration of $S$. ambofaciens genome has already led to the characterization of new gene clusters directing the biosynthesis of metabolites known to be produced by the strain (e.g. congocidine), to the identification of metabolites already characterized in other species but whose production by $S$. ambofaciens was not documented (e.g. kinamycins, antimycins), and to new gene clusters directing the biosynthesis of novel molecules (e.g. stambomycins). As the exploration continues, it is likely that it will result in the characterization of new clusters and molecules because several of the identified gene starter unit of the NPRS. b Chemical structure of antimycins identified in S. ambofaciens ATCC 23877 and general structure of the blastmycinone and butenolide compounds derived from antimycins

clusters have no characterized homologues. It is interesting to note that three secondary metabolites, kinamycins, stambomycins and antimycins, have great potential as antitumour drugs [25, 37, 43]. Therefore, the availability of the cluster sequence and the easy genetic manipulation of S. ambofaciens ATCC 23877 make this strain a promising tool for development of anticancer agents.

Several of the genome mined clusters are conserved in its close relative $S$. coelicolor $\mathrm{A} 3(2)$ allowing to propose a product associated with these clusters. For most of them, this has been experimentally demonstrated following the availability of the genome (e.g. the siderophores coelicheline and desferrioxamines [5]) or independently (e.g. the volatiles MIB, geosmin and albaflavenone [14]). Interestingly, genome comparison between $S$. ambofaciens ATCC 23877 and S. coelicolor A3(2) showed that the common clusters are not necessarily located within the conserved region, i.e. the core region which is highly syntenic through the genus and contains most of the essential genes [7] and the parts of the chromosomal arms which are conserved between the two species [13]. Thus, some are located within the specific extremities of the genome of the two species (in S. ambofaciens, these regions cover about $1.3 \mathrm{Mb}$ [13]). This is the case for the MIB and lantibiotic biosynthetic genes located in the left and right arm, respectively (Fig. 1). This probably reflects the plasticity of the Streptomyces genome which is prone to DNA rearrangements particularly in the terminal regions [42]. In contrast, specific clusters are not always located in the 
species-specific regions. Some correspond to genomic islands that interrupt the conserved regions. Thus, in $S$. ambofaciens ATCC 23877, seven secondary metabolite gene clusters, including the duplicated kinamycin cluster and the stambomycin and antimycin clusters, are located within the $1.3-\mathrm{Mb}$ specific extremities while six others, including the spiramycin and congocidine clusters, are encoded within conserved regions. In S. coelicolor A3(2), clusters such as those responsible for the biosynthesis of actinorhodin, undecylprodigiosin, coelimycin and CDA also correspond to specific genomic islands. These clusters would have a recent origin and probably result from recent acquisition by horizontal transfer events although one cannot exclude the possibility that, at least for some of them, they were once present in a Streptomyces species but have since been lost.

Genome sequence analysis has highlighted the potential of $S$. ambofaciens ATCC 23877 to produce natural products and has confirmed that it can be considered as a source of new bioactive molecules, as shown by the discovery of the stambomycins [37]. This potential has been determined thanks to bioinformatic tools such as antiSMASH [45] that allows efficient detection of secondary metabolite gene clusters belonging to a large range of classes or more specialized programs like NP.searcher [44] or NaPDos [76] mainly focusing on PKS and NRPS gene clusters. Nevertheless, S. ambofaciens likely has the capability to produce more natural products than those predicted by these approaches. Indeed, it is reasonable to consider that its genome contains genes or gene clusters directing the biosynthesis of products synthesized by still uncharacterized genes/enzymes. There are an increasing number of examples of products whose biosynthetic gene clusters could not have been detected by sequence comparison searches like the modified cyclic dipeptide alboursin [39] or the thiopeptide antibiotic thiostrepton [32]. Therefore, it is likely that $S$. ambofaciens and more generally the bacteria of the Streptomyces genus still contain a hidden treasure in terms of secondary metabolites and deserve our attention in the race for the discovery of novel bioactive compounds.

Acknowledgments This work was supported by the European Community under the ActinoGEN 6th framework programme (FP65224), the French National Research Agency through the Laboratory of Excellence ARBRE (ANR-12- LABXARBRE-01) and the Région Lorraine.

\section{References}

1. Aigle B, Bunet R, Corre C, Garénaux A, Hotel L, Huang S, Laureti L, Lautru S, Mendes MV, Nezbedova S, Nguyen HC, Song L, Weiser J, Challis G, Leblond P, Pernodet JL (2011) Genomeguided exploration of Streptomyces ambofaciens secondary metabolism. In: Dyson P (ed) Streptomyces, molecular biology and biotechnology. Caister Academic, Norfolk, pp 179-194

2. Aigle B, Corre C (2012) Waking up Streptomyces secondary metabolism by constitutive expression of activators or genetic disruption of repressors. Methods Enzymol 517:343-366. doi:10.1016/B978-0-12-404634-4.00017-6

3. Aigle B, Pang X, Decaris B, Leblond P (2005) Involvement of $\mathrm{AlpV}$, a new member of the Streptomyces antibiotic regulatory protein family, in regulation of the duplicated type II polyketide synthase alp gene cluster in Streptomyces ambofaciens. J Bacteriol 187(7):2491-2500. doi:10.1128/JB.187.7.2491-2500.2005

4. Baltz R (2007) Antimicrobials from actinomycetes: back to the future. Microbe 2(3):125-131

5. Barona-Gomez F, Lautru S, Francou FX, Leblond P, Pernodet JL, Challis GL (2006) Multiple biosynthetic and uptake systems mediate siderophore-dependent iron acquisition in Streptomyces coelicolor A3(2) and Streptomyces ambofaciens ATCC 23877. Microbiology 152(Pt 11):3355-3366. doi:10.1099/mic.0.29161-0

6. Barona-Gomez F, Wong U, Giannakopulos AE, Derrick PJ, Challis GL (2004) Identification of a cluster of genes that directs desferrioxamine biosynthesis in Streptomyces coelicolor M145. J Am Chem Soc 126(50):16282-16283. doi:10.1021/ja045774k

7. Bentley SD, Chater KF, Cerdeno-Tarraga AM, Challis GL, Thomson NR, James KD, Harris DE, Quail MA, Kieser H, Harper D, Bateman A, Brown S, Chandra G, Chen CW, Collins M, Cronin A, Fraser A, Goble A, Hidalgo J, Hornsby T, Howarth S, Huang CH, Kieser T, Larke L, Murphy L, Oliver K, O’Neil S, Rabbinowitsch E, Rajandream MA, Rutherford K, Rutter S, Seeger K, Saunders D, Sharp S, Squares R, Squares S, Taylor K, Warren T, Wietzorrek A, Woodward J, Barrell BG, Parkhill J, Hopwood DA (2002) Complete genome sequence of the model actinomycete Streptomyces coelicolor A3(2). Nature 417(6885):141-147. doi:10.1038/417141a

8. Bergmann S, Schumann J, Scherlach K, Lange C, Brakhage AA, Hertweck C (2007) Genomics-driven discovery of PKS-NRPS hybrid metabolites from Aspergillus nidulans. Nat Chem Biol 3(4):213-217. doi:10.1038/nchembio869

9. Bisang C, Long PF, Cortes J, Westcott J, Crosby J, Matharu AL, Cox RJ, Simpson TJ, Staunton J, Leadlay PF (1999) A chain initiation factor common to both modular and aromatic polyketide synthases. Nature 401(6752):502-505. doi:10.1038/46829

10. Bunet R, Mendes MV, Rouhier N, Pang X, Hotel L, Leblond P, Aigle B (2008) Regulation of the synthesis of the angucyclinone antibiotic alpomycin in Streptomyces ambofaciens by the autoregulator receptor AlpZ and its specific ligand. J Bacteriol 190(9):3293-3305. doi:10.1128/JB.01989-07

11. Bunet R, Song L, Mendes MV, Corre C, Hotel L, Rouhier N, Framboisier X, Leblond P, Challis GL, Aigle B (2011) Characterization and manipulation of the pathway-specific late regulator AlpW reveals Streptomyces ambofaciens as a new producer of kinamycins. J Bacteriol 193(5):1142-1153. doi:10.1128 /JB.01269-10

12. Chew WK, Segarra I, Ambu S, Mak JW (2012) Significant reduction of brain cysts caused by Toxoplasma gondii after treatment with spiramycin coadministered with metronidazole in a mouse model of chronic toxoplasmosis. Antimicrob Agents Chemother 56(4):1762-1768. doi:10.1128/AAC.05183-11

13. Choulet F, Aigle B, Gallois A, Mangenot S, Gerbaud C, Truong C, Francou FX, Fourrier C, Guerineau M, Decaris B, Barbe V, Pernodet JL, Leblond P (2006) Evolution of the terminal regions of the Streptomyces linear chromosome. Mol Biol Evol 23(12):2361-2369. doi:10.1093/molbev/ms1108

14. Citron CA, Gleitzmann J, Laurenzano G, Pukall R, Dickschat JS (2012) Terpenoids are widespread in actinomycetes: a correlation of secondary metabolism and genome data. ChemBioChem 13(2):202-214. doi:10.1002/cbic.201100641 
15. Cosar C, Ninet L, Pinnert-Sindico S, Preud'Homme J (1952) Trypanocide action of an antibiotic produced by a Streptomyces. C R Hebd Seances Acad Sci 234(14):1498-1499

16. Finlay AC, Hochstein FA, Sobin BA, Murphy FX (1951) Netropsin, a new antibiotic produced by a Streptomyces. J Am Chem Soc 73:341-343

17. Geistlich M, Losick R, Turner JR, Rao RN (1992) Characterization of a novel regulatory gene governing the expression of a polyketide synthase gene in Streptomyces ambofaciens. Mol Microbiol 6(14):2019-2029

18. Gerber N (1973) Volatile lactones from Streptomyces. Tetrahedron Lett 14(10):771-774

19. Gokhale RS, Hunziker D, Cane DE, Khosla C (1999) Mechanism and specificity of the terminal thioesterase domain from the erythromycin polyketide synthase. Chem Biol 6(2):117-125. doi:10.1016/S1074-5521(99)80008-8

20. Gomez-Escrabino J, Song L, Fox D, Yeo V, Bibb M, Challis G (2012) Structure and biosynthesis of the unusual polyketide alkaloid coelimycin P1, a metabolic product of the cpk gene cluster of Streptomyces coelicolor M145. Chem Sci 3:2716-2720

21. Gomez-Escribano JP, Bibb MJ (2012) Streptomyces coelicolor as an expression host for heterologous gene clusters. Methods Enzymol 517:279-300. doi:10.1016/B978-0-12-404634-4.00014-0

22. Gomez-Escribano JP, Bibb MJ (2013) Heterologous expression of natural product biosynthetic gene clusters in Streptomyces coelicolor: from genome mining to manipulation of biosynthetic pathways. J Ind Microbiol Biotechnol. doi:10.1007/ s10295-013-1348-5

23. Gould SJ (1997) Biosynthesis of the kinamycins. Chem Rev 97(7):2499-2510

24. Gould SJ, Hong ST, Carney JR (1998) Cloning and heterologous expression of genes from the kinamycin biosynthetic pathway of Streptomyces murayamaensis. J Antibiot (Tokyo) 51(1):50-57

25. Hasinoff BB, Wu X, Yalowich JC, Goodfellow V, Laufer RS, Adedayo O, Dmitrienko GI (2006) Kinamycins A and C, bacterial metabolites that contain an unusual diazo group, as potential new anticancer agents: antiproliferative and cell cycle effects. Anticancer Drugs 17(7):825-837

26. Juguet M, Lautru S, Francou FX, Nezbedova S, Leblond P, Gondry M, Pernodet JL (2009) An iterative nonribosomal peptide synthetase assembles the pyrrole-amide antibiotic congocidine in Streptomyces ambofaciens. Chem Biol 16(4):421-431. doi:10.1016/j.chembiol.2009.03.010

27. Kaiser S, Muller JJ, Froehlich PE, Baggio Gnoatto SC, Bergold AM (2013) From bacteria to antineoplastic: epothilones a successful history. Anticancer Agents Med Chem 13(7): 1057-1068

28. Kamra P, Gokhale RS, Mohanty D (2005) SEARCHGTr: a program for analysis of glycosyltransferases involved in glycosylation of secondary metabolites. Nucleic Acids Res 33 (Web Server issue):W220-W225. doi:10.1093/nar/gki449

29. Karray F, Darbon E, Nguyen HC, Gagnat J, Pernodet JL (2010) Regulation of the biosynthesis of the macrolide antibiotic spiramycin in Streptomyces ambofaciens. J Bacteriol 192(21):5813-5821. doi:10.1128/JB.00712-10

30. Karray F, Darbon E, Oestreicher N, Dominguez H, Tuphile K, Gagnat J, Blondelet-Rouault MH, Gerbaud C, Pernodet JL (2007) Organization of the biosynthetic gene cluster for the macrolide antibiotic spiramycin in Streptomyces ambofaciens. Microbiology 153(Pt 12):4111-4122. doi:10.1099/mic.0.2007/009746-0

31. Keatinge-Clay AT (2007) A tylosin ketoreductase reveals how chirality is determined in polyketides. Chem Biol 14(8):898-908. doi:10.1016/j.chembiol.2007.07.009

32. Kelly WL, Pan L, Li C (2009) Thiostrepton biosynthesis: prototype for a new family of bacteriocins. J Am Chem Soc 131(12):4327-4334. doi:10.1021/ja807890a
33. Kim BS, Cropp TA, Beck BJ, Sherman DH, Reynolds KA (2002) Biochemical evidence for an editing role of thioesterase II in the biosynthesis of the polyketide pikromycin. J Biol Chem 277(50):48028-48034. doi:10.1074/jbc.M207770200

34. Kuhstoss S, Huber M, Turner JR, Paschal JW, Rao RN (1996) Production of a novel polyketide through the construction of a hybrid polyketide synthase. Gene 183(1-2):231-236

35. Kuscer E, Coates N, Challis I, Gregory M, Wilkinson B, Sheridan $\mathrm{R}$, Petkovic H (2007) Roles of rapH and rapG in positive regulation of rapamycin biosynthesis in Streptomyces hygroscopicus. J Bacteriol 189(13):4756-4763. doi:10.1128/JB.00129-07

36. Kwan DH, Sun Y, Schulz F, Hong H, Popovic B, Sim-Stark JC, Haydock SF, Leadlay PF (2008) Prediction and manipulation of the stereochemistry of enoylreduction in modular polyketide synthases. Chem Biol 15(11):1231-1240. doi:10.1016/j.chembiol.2008.09.012

37. Laureti L, Song L, Huang S, Corre C, Leblond P, Challis GL, Aigle B (2011) Identification of a bioactive 51-membered macrolide complex by activation of a silent polyketide synthase in Streptomyces ambofaciens. Proc Natl Acad Sci U S A 108(15):6258-6263. doi:10.1073/pnas.1019077108

38. Lautru S, Deeth RJ, Bailey LM, Challis GL (2005) Discovery of a new peptide natural product by Streptomyces coelicolor genome mining. Nat Chem Biol 1(5):265-269. doi:10.1038/nchembio731

39. Lautru S, Gondry M, Genet R, Pernodet JL (2002) The albonoursin gene cluster of $S$ noursei biosynthesis of diketopiperazine metabolites independent of nonribosomal peptide synthetases. Chem Biol 9(12):1355-1364

40. Lautru S, Song L, Demange L, Lombes T, Galons H, Challis GL, Pernodet JL (2012) A sweet origin for the key congocidine precursor 4-acetamidopyrrole-2-carboxylate. Angew Chem Int Ed Engl 51(30):7454-7458. doi:10.1002/anie.201201445

41. Leben C, Keitt G (1948) An antibiotic substance active against certain phytopathogens. Phytopathology 38:899-906

42. Leblond P, Demuyter P, Simonet JM, Decaris B (1991) Genetic instability and associated genome plasticity in Streptomyces ambofaciens: pulsed-field gel electrophoresis evidence for large DNA alterations in a limited genomic region. J Bacteriol 173(13):4229-4233

43. Lee SJ, Kim EA, Song KS, Kim MJ, Lee DH, Kwon TK, Lee TJ (2012) Antimycin A sensitizes cells to TRAIL-induced apoptosis through upregulation of DR5 and downregulation of c-FLIP and Bcl-2. Int J Oncol 41(4):1425-1430. doi:10.3892/ijo2012.1575

44. Li MH, Ung PM, Zajkowski J, Garneau-Tsodikova S, Sherman DH (2009) Automated genome mining for natural products. BMC Bioinformatics 10:185. doi:10.1186/1471-2105-10-185

45. Medema MH, Blin K, Cimermancic P, de Jager V, Zakrzewski P, Fischbach MA, Weber T, Takano E, Breitling R (2011) antiSMASH: rapid identification, annotation and analysis of secondary metabolite biosynthesis gene clusters in bacterial and fungal genome sequences. Nucleic Acids Res 39 (Web Server issue):W339-W346. doi:10.1093/nar/gkr466

46. Medema MH, Trefzer A, Kovalchuk A, van den Berg M, Muller U, Heijne W, Wu L, Alam MT, Ronning CM, Nierman WC, Bovenberg RA, Breitling R, Takano E (2010) The sequence of a 1.8-mb bacterial linear plasmid reveals a rich evolutionary reservoir of secondary metabolic pathways. Genome Biol Evol 2:212224. doi:10.1093/gbe/evq013

47. Mochizuki S, Hiratsu K, Suwa M, Ishii T, Sugino F, Yamada K, Kinashi H (2003) The large linear plasmid pSLA2-L of Streptomyces rochei has an unusually condensed gene organization for secondary metabolism. Mol Microbiol 48(6):1501-1510

48. Moore JM, Bradshaw E, Seipke RF, Hutchings MI, McArthur M (2012) Use and discovery of chemical elicitors that stimulate biosynthetic gene clusters in Streptomyces bacteria. Methods Enzymol 517:367-385. doi:10.1016/B978-0-12-404634-4.00018-8 
49. Nguyen HC, Darbon E, Thai R, Pernodet JL, Lautru S (2013) Post-PKS tailoring steps of the spiramycin macrolactone ring in Streptomyces ambofaciens. Antimicrob Agents Chemother 57(8):3836-3842. doi:10.1128/AAC.00512-13

50. Nguyen HC, Karray F, Lautru S, Gagnat J, Lebrihi A, Huynh TD, Pernodet JL (2010) Glycosylation steps during spiramycin biosynthesis in Streptomyces ambofaciens: involvement of three glycosyltransferases and their interplay with two auxiliary proteins. Antimicrob Agents Chemother 54(7):2830-2839. doi:10.1128/ AAC.01602-09

51. O'Hara KA, Dmitrienko GI, Hasinoff BB (2010) Kinamycin F downregulates cyclin D3 in human leukemia K562 cells. Chem Biol Interact 184(3):396-402. doi:10.1016/j.cbi.2010.01.013

52. Ochi K, Hosaka T (2013) New strategies for drug discovery: activation of silent or weakly expressed microbial gene clusters. Appl Microbiol Biotechnol 97(1):87-98. doi:10.1007/ s00253-012-4551-9

53. Ochi K, Tanaka Y, Tojo S (2013) Activating the expression of bacterial cryptic genes by rроB mutations in RNA polymerase or by rare earth elements. J Ind Microbiol Biotechnol. doi:10.1007/ s10295-013-1349-4

54. Pang X, Aigle B, Girardet JM, Mangenot S, Pernodet JL, Decaris B, Leblond P (2004) Functional angucycline-like antibiotic gene cluster in the terminal inverted repeats of the Streptomyces ambofaciens linear chromosome. Antimicrob Agents Chemother 48(2):575-588

55. Parajuli N, Basnet DB, Chan Lee H, Sohng JK, Liou K (2004) Genome analyses of Streptomyces peucetius ATCC 27952 for the identification and comparison of cytochrome P450 complement with other Streptomyces. Arch Biochem Biophys 425(2):233241. doi:10.1016/j.abb.2004.03.011

56. Pinnert-Sindico S (1954) Une nouvelle espèce de Streptomyces productrice d'antibiotiques: Streptomyces ambofaciens $\mathrm{n}$. sp. caractères culturaux. Ann Inst Pasteur (Paris) 87(6):702-707

57. Poulet PP, Duffaut D, Barthet P, Brumpt I (2005) Concentrations and in vivo antibacterial activity of spiramycin and metronidazole in patients with periodontitis treated with high-dose metronidazole and the spiramycin/metronidazole combination. J Antimicrob Chemother 55(3):347-351. doi:10.1093/jac/dki013

58. Rebets Y, Brotz E, Tokovenko B, Luzhetskyy A (2013) Actinomycetes biosynthetic potential: how to bridge in silico and in vivo? J Ind Microbiol Biotechnol. doi:10.1007/s10295-013-1352-9

59. Reid R, Piagentini M, Rodriguez E, Ashley G, Viswanathan N, Carney J, Santi DV, Hutchinson CR, McDaniel R (2003) A model of structure and catalysis for ketoreductase domains in modular polyketide synthases. Biochemistry 42(1):72-79. doi:10.1021/ bi0268706

60. Richardson MA, Kuhstoss S, Huber ML, Ford L, Godfrey O, Turner JR, Rao RN (1990) Cloning of spiramycin biosynthetic genes and their use in constructing Streptomyces ambofaciens mutants defective in spiramycin biosynthesis. J Bacteriol 172(7):3790-3798

61. Richardson MA, Kuhstoss S, Solenberg P, Schaus NA, Rao RN (1987) A new shuttle cosmid vector, pKC505, for streptomycetes: its use in the cloning of three different spiramycinresistance genes from a Streptomyces ambofaciens library. Gene 61(3):231-241
62. Riclea R, Aigle B, Leblond P, Schoenian I, Spiteller D, Dickschat JS (2012) Volatile lactones from streptomycetes arise via the antimycin biosynthetic pathway. ChemBioChem 13(11):1635-1644. doi:10.1002/cbic. 201200260

63. Schoenian I, Paetz C, Dickschat JS, Aigle B, Leblond P, Spiteller D (2012) An unprecedented 1,2-shift in the biosynthesis of the 3-aminosalicylate moiety of antimycins. ChemBioChem 13(6):769-773. doi:10.1002/cbic.201200033

64. Seipke RF, Barke J, Brearley C, Hill L, Yu DW, Goss RJ, Hutchings MI (2011) A single Streptomyces symbiont makes multiple antifungals to support the fungus farming ant Acromyrmex octospinosus. PLoS ONE 6(8):e22028. doi:10.1371/ journal.pone. 0022028

65. Stumpp T, Himbert S, Altenbuchner J (2005) Cloning of the netropsin resistance genes from Streptomyces flavopersicus NRRL 2820. J Basic Microbiol 45(5):355-362. doi:10.1002/j obm.200410529

66. Tener G, Merlin Bumpus F, Dunshee B, Strong F (1953) The structure of antimycin A. II. Degradation studies. J Am Chem Soc 75(5):1100-1104

67. Tener G, van Tamelen E, Strong F (1953) The chemistry of antimycin A. III. Structure of the antimycic acid. J Am Chem Soc 75(15):3623-3625

68. van Tamelen E, Strong F, Quarck U (1959) The chemistry of antimycin A. IV. Study of the structure of antimycin lactone. J Am Chem Soc 81(3):750-751

69. van Wezel GP, McDowall KJ (2011) The regulation of the secondary metabolism of Streptomyces: new links and experimental advances. Nat Prod Rep 28(7):1311-1333. doi:10.1039/ c1np00003a

70. Welker M, Dittmann E, von Dohren H (2012) Cyanobacteria as a source of natural products. Methods Enzymol 517:23-46. doi:10.1016/B978-0-12-404634-4.00002-4

71. Wilkinson CJ, Hughes-Thomas ZA, Martin CJ, Bohm I, Mironenko T, Deacon M, Wheatcroft M, Wirtz G, Staunton J, Leadlay PF (2002) Increasing the efficiency of heterologous promoters in actinomycetes. J Mol Microbiol Biotechnol 4(4):417-426

72. Wilson DJ, Xue Y, Reynolds KA, Sherman DH (2001) Characterization and analysis of the PikD regulatory factor in the pikromycin biosynthetic pathway of Streptomyces venezuelae. J Bacteriol 183(11):3468-3475. doi:10.1128/JB.183.11.3468-3475.2001

73. Yadav G, Gokhale RS, Mohanty D (2003) Computational approach for prediction of domain organization and substrate specificity of modular polyketide synthases. J Mol Biol 328(2):335-363

74. Yadav G, Gokhale RS, Mohanty D (2003) SEARCHPKS: a program for detection and analysis of polyketide synthase domains. Nucleic Acids Res 31(13):3654-3658

75. Zhu H, Sandiford SK, van Wezel GP (2013) Triggers and cues that activate antibiotic production by actinomycetes. J Ind Microbiol Biotechnol. doi:10.1007/s10295-013-1309-z

76. Ziemert N, Podell S, Penn K, Badger JH, Allen E, Jensen PR (2012) The natural product domain seeker NaPDoS: a phylogeny based bioinformatic tool to classify secondary metabolite gene diversity. PLoS ONE 7(3):e34064. doi:10.1371/ journal.pone.0034064 